Methodology Material and methods: Case-control study comprised patients with ovarian endometriosis $(n=47)$, deep infiltrating endometriosis $(\mathrm{DIE})(\mathrm{n}=14)$, endometriosis in caesarean scar $(n=30)$, as well as patients with clear cell and endometrioid ovarian cancer $(n=26)$. For control group normal ovarian tissue from patients operated on for benign uterine pathology $(n=33)$ and high grade ovarian cancer (HGOC) samples $(n=29)$ were obtained. Total RNA was isolated from 1-2 tissue slices from archival formalin-fixed paraffin embedded (FFPE) blocks using the High Pure FFPET Isolation Kit (Roche). miRNA expression was first screened using TaqMan ${ }^{\circledR}$ Human MicroRNA Array A and B (Applied Biosystems). The expression levels of 754 human miRNA genes were assessed firstly in the groups of patients with EAOC $(n=10)$ and healthy controls $(n=10)$. MiRNAs with altered expression profiles were then chosen for further investigations. Quantification of these selected miRNA was done using TaqMan Advanced MicroRNA Assays (Applied Biosystems). The miRNA level was calculated as $2^{-\Delta \Delta} \mathrm{C} t$, while relative expression analysis of the examined gene was presented as an $n$-fold change in gene expression normalized to a reference gene relative to the control.

Result(s)* Several miRNAs were highly dysregulated between healthy ovarian tissue and EAOC tissue: hsa-miR-1-3p, hsamiR-31-3p, hsa-miR-125b-1-3p, hsa-miR-200b, miR548d. Validation revealed that the level of all tested miRNAs was only slightly higher in endometrial cysts comparing to normal ovarian tissue, but significantly higher in DIE foci and all types of ovarian cancer, including HGOC. The only miRNA that were able to discriminate between EAOC and HGOC was miR-1$3 \mathrm{p}$ which showed high expression in EAOC and lack of expression in HGOC.

Conclusion* The same pattern of miRNA expression in DIE and EAOC, but not in endometrial cysts, could help to predict epigenetic changes that may be responsible for carcionogenesis in endometriosis foci.

\section{THE ROLE OF NEOADJUVANT CHEMOTHERAPY IN THE TREATMENT OF IIIC-IVA STAGE EPITHELIAL OVARIAN CANCER - A SINGLE CENTER EXPERIENCE}

S Molnár*, LÉ Vas, E Maka, R Lampé, B Vida, Z Krasznai. University of Debrecen, Department of Obstetrics and Gynecology, Debrecen, Hungary

\subsection{6/ijgc-2021-ESG0.423}

Introduction/Background* Ovarian cancer is the 7th most common malignancy among women and the leading cause of gynecologic cancer death. The most important prognostic factor of the disease is optimal debulking surgery (R0) with no macroscopic residual disease. Achieving optimal result is a challenging duty in advanced stage (FIGO IIIC-IV). Based on previous studies neoadjuvant chemotherapy (NAC) can help to improve the optimally debulked ratio of this population with non-inferior survival outcome. The aim of our study was to evaluate the effectiveness of NAC among primarily inoperable patients. The focus was not only on survival outcome but on cost effectiveness (need for transfusion, hospitalization, ICU admission, medication demand, etc.).

Methodology Between 2015-2018 112 debulking surgeries were performed on stage FIGO IIIC-IV ovarian cancer patients. The cases were divided into potentially operable and inoperable group based on preoperative imaging, tumor marker levels according to our institutional protocol. In special situation where operability was not obvious, diagnostic laparoscopy was done to categorize patients. The peri-, intra-, postoperative reports and survival data was collected.

Result(s)* Complete tumor reduction was performed in 63 cases while in 49 cases only partial tumor reduction was achieved. Median progression-free survival did not differ significantly between patients who underwent primary or interval debulking surgery (PDS $=12$ months, IDS $=11.2$ months, $p$ $=0.264)$. The rate of R0 resection was higher after NAC, but not significantly $(37.9 \%$ vs. $54.2 \%, \mathrm{p}=0.179)$. There was no significant difference in survival of patients who successfully underwent complete tumor reduction, despite the fact that the "inoperable" group treated with NAC had a worse prognosis $(p=0.264)$. The cost-effectiveness was comparable between groups, the hospital stay and transfusion demand was favourable in neoadjuvant group.

Conclusion* The rate of optimal tumor reduction can be improved not only by increasing radicality but also by applying appropriate patient selection criteria. Neoadjuvant treatment according to the current recommendations is applicable in the inoperable group, in accordance with the protocol we use. The survival data of patients who have undergone complete tumor reduction after NAC was not inferior to those who went through primary debulking.

\section{CORRELATION BETWEEN CT, INTRA-OPERATIVE ASSESSMENT AND FINAL HISTOPATHOLOGY IN DETECTING DIAPHRAGMATIC DISEASE IN ADVANCED OVARIAN CANCER. 61 CASES}

${ }^{1} S$ Addley*, 'A Bali, ${ }^{1} V$ Asher, ${ }^{1} S$ Abdul, ${ }^{2}$ R Kirke, ${ }^{1}$ A Phillips. 'University Hospitals of Derby and Burton NHS Foundation Trust, Gynaecology Oncology; ${ }^{2}$ University Hospitals of Derby and Burton NHS Foundation Trust, Radiology

\subsection{6/ijgc-2021-ESG0.424}

Introduction/Background* Whilst CT scanning is well established in the pre-operative staging of tubo-ovarian or primary peritoneal malignancy, studies suggest CT may be a poor predictor of diaphragmatic involvement.

Methodology All patients who underwent diaphragmatic surgery for stage III/V tubo-ovarian or primary peritoneal carcinoma in a cancer centre between 2008-2020 were identified. Pre-operative CT, operation notes and histopathology reports were reviewed retrospectively.

Result(s)* 61 patients were identified. In 82.0\% ( $\mathrm{n}=50)$, no diaphragmatic disease was identified on pre-operative CT. This was corroborated by negative histopathology in 6 cases equating to $9.8 \%$ true negatives, $72.1 \%$ false negatives and negative predictive value of $12 \%$. Of those with diaphragmatic disease identified radiologically $(n=11)$, this was confirmed on histopathology in 10 cases - equating to $16.3 \%$ true positives, $1.6 \%$ false positives and positive predictive value of $90.9 \%$. Our data demonstrates a sensitivity of $18.5 \%$ and specificity of $85.7 \%$ for CT in diaphragmatic assessment.

Intra-operative findings were suspicious for diaphragmatic involvement in all cases and confirmed on histopathology in $90.1 \%(\mathrm{n}=55)$. In $6(9.8 \%)$ patients, however, histopathology returned negative. Statistical analysis of this data was limited by the inclusion criteria - but can be interpreted as $90.2 \%$ true positives, $9.8 \%$ false positives and positive predictive 
value of $90.2 \%$ for intra-operative detection of diaphragmatic disease

Conclusion* Whilst it is accepted that CT is a poor predictor of diaphragmatic disease, we suggest our figures may be

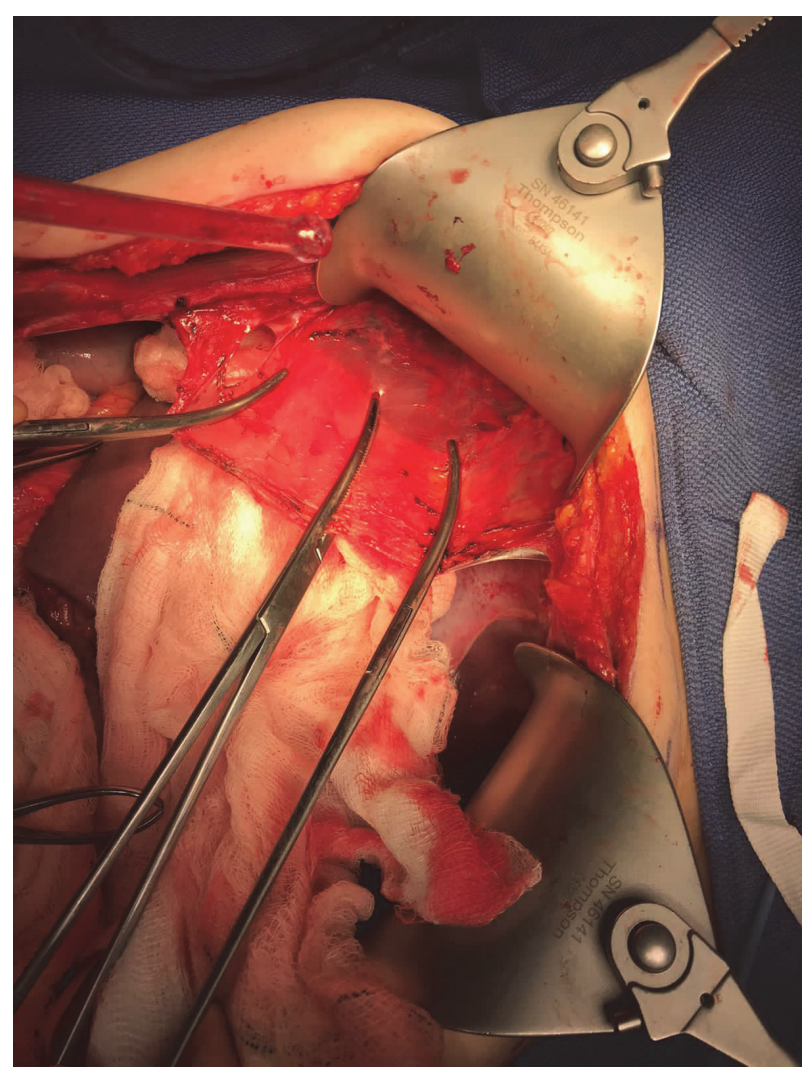

Abstract 543 Figure 1

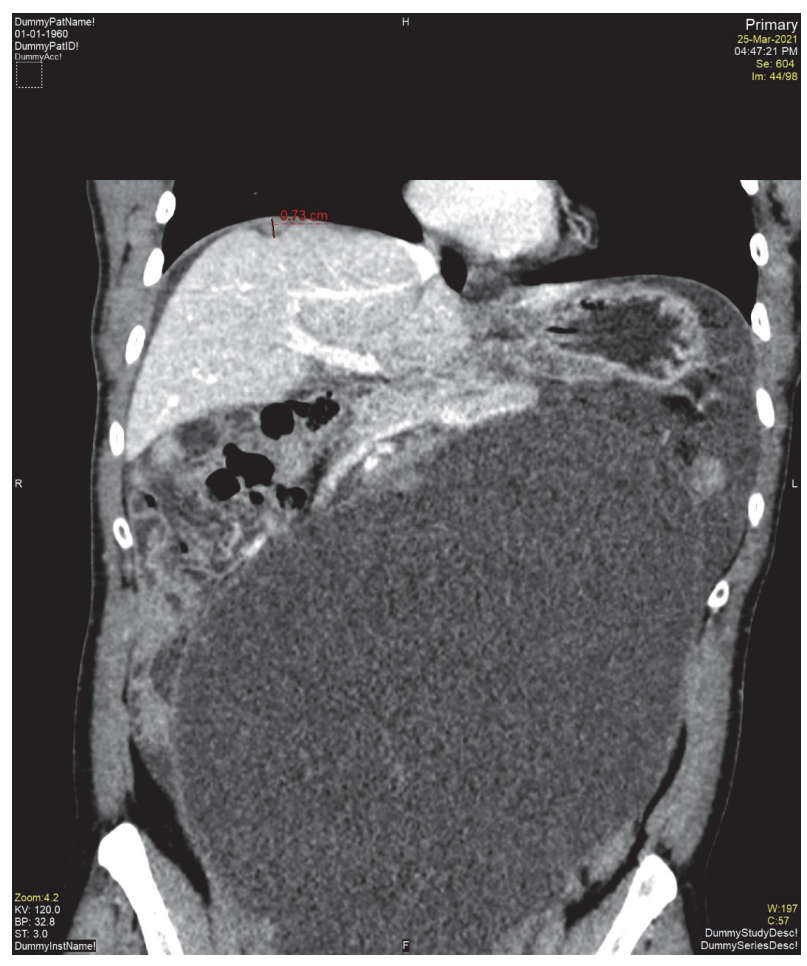

Abstract 543 Figure 2 additionally compounded by a local radiological focus on identification of surgical stopping-points in the context of a unit with a well-established ultra-radical service and experience of diaphragmatic surgery. Gynaecological-oncologists should, however, remain mindful of the limitations of CT and hence approach all relevant cases with the anticipation of encountering diaphragmatic disease.

\section{DIAGNOSTIC AND THERAPEUTIC APPROACHES FOR PATIENTS WITH A STIC LESION - RESULTS OF THE AGO OVAR QUESTIONNAIRE BASED SURVEY IN GERMANY}

1J Van der Ven*, 'V Linz, 'S Krajnak, ${ }^{1} \mathrm{~K}$ Anic, ${ }^{1} \mathrm{R}$ Schwab, ${ }^{2} \mathrm{~S}$ Kommoss, ${ }^{3} \mathrm{~B}$ Schmalfeldt, 4J Sehouli, ${ }^{1} \mathrm{~A}$ Hasenburg, ${ }^{1} \mathrm{M}$ Battista. 'Unimedizin Mainz, Klinik und Poliklinik für Geburtshilfe und Frauengesundheit; '2Universitätsklinikum Tübingen, Department für Frauengesundheit; ${ }^{3}$ Universitätsklinikum Hamburg-Eppendorf, Klinik und Poliklinik für Gynäkologie; ${ }^{4}$ Charité Berlin, Klinik für Gynäkologie mit Zentrum für onkologische Chirurgie

\subsection{6/ijgc-2021-ESG0.425}

Introduction/Background* Despite growing understanding of the carcinogenesis of high-grade serous ovarian cancer and its precursor lesion serous tubal intraepithelial carcinoma (STIC), there is a lack of evidence based recommendations for the clinical management of patients with a STIC lesion.

Methodology We created 23 questions to explore the experience with STIC patients and the diagnostical, surgical and histopathological approaches and used SoSci Survey to host the questionnaire. We invited all German directors of gynecological departments to participate.

Result(s)* 550 colleagues were invited. 131 questionnaires (response rate $24.3 \%$ ) were returned and included in this survey. $45.8 \%$ of the respondents treated one to three STIC patients. $76.0 \%$ of the participants performed opportunistic bilateral salpingectomies during other gynecological interventions. Most of the participants requested the SEE-FIM protocol from their pathologists since 2017. It was used by $54.2 \%$ for prophylactic salpingectomies, by $28.1 \%$ for both prophylactic and opportunistic salpingectomies and by $17.7 \%$ for neither of both. In a case of a STIC lesion 58.8\%, 2.4\%, 37.6\% of participants used the laparoscopic, transvers- or longitudinal laparotomic approach, respectively. The respondents performed a hysterectomy, bilateral ovarectomy or affected side ovarectomy in pre- and postmenopausal patients in $25.6 \%(54.6 \%)$, $24.42(88.4 \%)$ and $50.0 \%(4.6 \%)$, respectively (all p-values $>0.001$ ). Omentectomy, pelvic and para-aortic lymphadenectomy were performed in pre- and postmenopausal women in $60.5 \%$ (63.9\%), 9.30\% (11.6\%) and 9.3\% (11.6\%) (all p-values <0.05).

Conclusion* This survey highlights significant inconsistency in the management of patients with a STIC lesion. Further studies are urgently warranted to elucidate the clinical impact and the necessary therapeutic approach of STIC lesions.

\section{UTERINE SEROUS CARCINOMA MIMICKING ADVANCED OVARIAN CANCER. ARE THERE CLUES TO PRE- OPERATIVE DIAGNOSTIC DIFFERENTIATION? A MINI CASE SERIES}

S Addley*, A Bali, V Asher, S Abdul, A Phillips. University Hospitals of Derby and Burton NHS Foundation Trust, Gynaecology Oncology, UK

10.1136/ijgc-2021-ESG0.426 